\title{
Upregulation of ADAMTS-7 and downregulation of COMP are associated with aortic aneurysm
}

\author{
WEI QIN*, YIDE CAO* , LIANGPENG LI, WEN CHEN and XIN CHEN \\ Department of Cardiothoracic Surgery, Nanjing First Hospital, Nanjing \\ Medical University, Nanjing, Jiangsu 210006, P.R. China
}

Received December 6, 2016; Accepted June 29, 2017

DOI: $10.3892 / \mathrm{mmr} .2017 .7293$

\begin{abstract}
Aortic aneurysm (AA) remains a fatal condition with high rates of morbidity and mortality, and the associated underlying mechanism influencing its pathology remains to be elucidated. A disintegrin and metalloproteinase with thrombospondin motifs (ADAMTS)-7 has previously been demonstrated to be involved in the pathogenesis of vascular atherosclerosis via degradation of cartilage oligomeric matrix protein (COMP). The ADAMTS-7/COMP pathway may therefore act as a potential therapeutic target for vascular disorders. To the best of the author's knowledge, the present study aimed to investigate for the first time, the expression of ADAMTS-7 and COMP in human AA. Human aortic aneurysm samples were collected from patients with AA ( $n=24)$, and ascending aorta control samples were harvested from dilated cardiomyopathy patients who underwent heart transplantation $(n=18)$. Expression levels of ADAMTS-7 and matrix metalloproteinase-9 were significantly increased in the AA group, as detected by immunohistochemistry $(\mathrm{P}<0.05)$. The COMP protein level was markedly decreased in the AA group when compared with the control group, as demonstrated via immunohistochemistry and western blot analysis $(\mathrm{P}<0.05)$. The findings suggest that upregulation of ADAMTS-7 and downregulation of COMP are associated with induction of human AA. ADAMTS-7/COMP pathway may provide therefore act as a potential therapeutic target in human AA for efficient, optimal treatment interventions in the future.
\end{abstract}

\section{Introduction}

The primary disease processes affecting the aorta are aortic aneurysms (AAs) and aortic dissections, which have high rates of mortality and morbidity. Data are limited with regard to

Correspondence to: Dr Xin Chen, Department of Cardiothoracic Surgery, Nanjing First Hospital, Nanjing Medical University, 68 Changle Road, Nanjing, Jiangsu 210006, P.R. China

E-mail: chenxinnjdyyy@163.com

${ }^{*}$ Contributed equally

Key words: aortic aneurysm, a disintegrin and metalloproteinase with thrombospondin motifs 7 , cartilage oligomeric matrix protein the pathologies of AA, characterized by disorders and loss of vascular smooth muscle cells (VSMCs), fragmentation of elastic fibers, degradation of extracellular matrix (ECM) and infiltration of macrophages and $\mathrm{T}$ lymphocytes (1). The exact underlying mechanisms influencing the occurrence of AA remain to be fully elucidated.

A disintegrin and metalloproteinase with thrombospondin motifs (ADAMTS), belonging to the metalloproteinase family, is important in bone formation and arterial/cardiac remodeling (2-4). Various ADAMTS family members are highly expressed in human carotid lesions and advanced coronary atherosclerotic plaques, including ADAMTS-1, -4, $-5,-7$ and -8 (5-9). In addition, various findings suggested that the ADAMTS-7-associatedadvancedatherosclerosis may be associated with degradation of cartilage oligomeric matrix protein (COMP), a protein secreted by VSMCs $(6,10,11)$. COMP was originally observed as a component of cartilage and tendons. Mutations in the COMP gene may induce pseudoachondroplasia and multiple epiphyseal dysplasia $(12,13)$. Oldberg et al (14) revealed that there was a low expression of COMP mRNA in rat aortic tissue however, the group did not pursue this finding further. Previous studies $(15,16)$ have also demonstrated that COMP participates in some pathophysiologies, including vascular calcification, atherosclerosis and neointima formation post-injury. In the present study, ADAMTS-7/COMP was considered to participate in the pathogenesis of vascular disorders and may therefore act as a novel and potential therapeutic target for the treatment of atherosclerosis and vascular disorders. The present study investigated the expression levels of ADAMTS-7 and COMP in human AA samples and aimed to identify the associated underlying mechanism.

\section{Materials and methods}

Aortic tissue. All the patients gave informed consent for aortic tissue harvesting and testing, and all experiments were approved by the ethical committee of Nanjing Medical University and Nanjing First Hospital (Nanjing, China). Full-thickness aortic wall samples were collected from the ascending proportion of the aorta, from patients with AA $(n=24 ; 16$ male, 8 female; average age, 57.25 \pm 9.29 ; those with pseudoaneurysms were excluded). None of the aforementioned patients had a known genetic disorder resulting 
in the aortic disease or a family history of AA. Control samples were obtained from 18 patients with dilated cardiomyopathy who received heart transplantation surgery $(n=18$; 14 male, 4 female; average age $47.67 \pm 10.58$ ). All participants in the present study were recruited at the Department of Cardiothoracic Surgery, Nanjing First Hospital (Nanjing, China) between January 2015 and January 2016. Samples were immediately transferred to the laboratory and divided into two sample groups. The first sample group was used for protein extraction for western blotting, and the second for staining and immunohistochemistry.

Hematoxylin and eosin $(H \& E)$ and masson staining. Aortas were removed and immediately immersed in $4 \%$ neutral phosphate-buffered paraformaldehyde for $12 \mathrm{~h}$ at room temperature. The aortas were then embedded in paraffin following dehydration with an ethanol gradient and sectioned $(5 \mu \mathrm{m})$. The sections were rehydrated in graded alcohol, then stained firstly with hematoxylin for $10 \mathrm{~min}$, then eosin for 2 min all at room temperature. For masson staining, the sections were rehydrated in graded alcohol, then stained with masson composite dyeing fluid for $5 \mathrm{~min}$, followed by phosphomolybdic acid for $5 \mathrm{~min}$, then aniline blue for $5 \mathrm{~min}$ and finally ethanol hydrochloride differentiating solution for $30 \mathrm{sec}$; all staining procedures were performed at room temperature. The morphological alterations and fibrosis in the aortas were observed under a light microscope.

Immunohistochemical staining. Tissues collected for morphological analysis with aneurysm aortas were prepared as $5 \mu \mathrm{m}$ thick serial paraffin-embedded sections following heating at $65^{\circ} \mathrm{C}$ for $1 \mathrm{~h}$, which were rehydrated in graded alcohol and then washed with $1 \%$ phosphate buffer saline. The sections were treated with $3 \%$ hydrogen peroxide for 15 min to block endogenous peroxidase activity and incubated at room temperature in imported goat serum (catalog no. ZLI-9022; Beijing Zhongshan Jinqiao Biotechnology Co., Ltd., Beijing, China) for $1.5 \mathrm{~h}$ to prevent non-specific binding of antibodies. The sections were then incubated at $4^{\circ} \mathrm{C}$ separately for $14 \mathrm{~h}$ with antibodies against $\alpha$-smooth muscle actin (SMA; 1:400; catalog no. A2547; Sigma-Aldrich; Merck KGaA, Darmstadt, Germany), matrix metalloproteinase (MMP-9; 1:500; catalog no. ab38898; Abcam, Cambridge, UK), COMP (1:50; catalog no. ab11056; Abcam) and ADAMTS-7 (1:200; catalog no. ab203027; Abcam). Following this, sections were then incubated with horseradish peroxidase (HRP)-conjugated goat anti-rabbit or anti-mouse IgG antibodies (using the manufacturer's dilution; catalog nos. KIT-5004 and KIT-5001, respectively; Fuzhou Maixin Biotechnology Development, Co., Ltd., Fuzhou, China) for $15 \mathrm{~min}$ at room temperature in a humidified box. The signal of each antibody was developed using the substrate diaminobenzidine (catalog no. ZLI-9018; Beijing Zhongshan Jinqiao Biotechnology Co., Ltd). Sections were counterstained at room temperature with hematoxylin for $10 \mathrm{~min}$, and photomicrographs were taken by a ZEISS SCOPE.A1 camera (ZEISS GmbH, Jena, Germany). The results of immunohistochemistry were evaluated by Rajkumar score (17), in which the brown chromogen intensity was scored (range, 0-3). The average score of every section was assessed by two independent observers and used for later comparison.
Western blot analysis. Human aneurysm aortas were lysed in lysis buffer containing $10 \mathrm{ml}$ phosphatase inhibitors, $1 \mathrm{ml}$ protease inhibitors and $5 \mathrm{ml}$ PMSF per milliliter (catalog no. KGP250; Nanjing KeyGen BioTech Co., Ltd., Nanjing, China) and sonicated to extract total proteins. Whole tissue lysates were then centrifuged at $8,050 \mathrm{xg}$ for $15 \mathrm{~min}$ at $4^{\circ} \mathrm{C}$. Total proteins were quantified using the Bicinchoninic Acid method. Total proteins $(50 \mu \mathrm{g} / \mathrm{lane})$ were separated by $8 \%$ SDS-PAGE. The proteins were transferred to polyvinylidene fluoride (PVDF) membranes (EMD Millipore, Billerica, MA, USA), which were washed twice in Tris-buffered saline (TBS) with Tween ${ }^{\circledR}$ diluted 1:1,000 (TBST; Promega Corporation, Madison, WI, USA), for 10 min each time, and blocked at room temperature with TBST containing 5\% non-fat milk powder for $1 \mathrm{~h}$. The membranes were probed with the following primary antibodies in TBST plus 5\% non-fat milk overnight at $4^{\circ} \mathrm{C}$ : anti-COMP $(1: 1,500$; catalog no. ab11056; Abcam) and HRP-conjugated monoclonal mouse anti-GAPDH (1:5,000; catalog no. KC-5G5; Zhejiang Kangchen Biotech Co., Ltd., Wuhan, China). The following day, PVDF membranes were washed with TBST four times for 10 min each. Subsequently, the PVDF membranes were incubated with appropriately diluted HRP-conjugated goat anti-mouse secondary antibodies (1:5,000; catalog no. bs-0296G-HRP; BIOSS, Beijing, China) at room temperature for $1 \mathrm{~h}$. Specific proteins were detected using an immobilon western chemiluminescent HRP substrate (catalog no. WBKLS0100; EMD Millipore) and captured on Hyperfilm (GE Healthcare Life Sciences, Little Chalfont, UK). The results were then analyzed using Image $\mathbf{J}$ software v1.37c (National Institutes of Health, USA) for semi-quantitation of the mean gray value for each blot. Following this, the SPSS v13.0 statistical software (SPSS, Inc., Chicago, IL, USA) was used to perform independent-samples t-test to detect the differences between the aneurysm aortas and the control samples.

Statistical analysis. Data are expressed as the mean \pm standard deviation and analyses were performed using SPSS v13.0 statistical software (SPSS, Inc.). For the comparison of group means, a check was first made for similar variances: If this was passed then an unpaired two sample two-tailed Student's t-test was carried out; if the variances were significantly different, then an unpaired two sample two-tailed t-test with Welch's correction was used for these 2 groups. $\mathrm{P}<0.05$ was considered to indicate a statistically significant difference.

\section{Results}

Fracture of VSMCs and degeneration of collagen in the media of aneurysm aortas. As presented in Figs. 1 and 2, in the aortic media of control samples, almost all VSMCs demonstrated an orderly pattern of immunoreactive $\alpha$-SMA and formed homogeneous layers separated by elastic laminae in an orderly pattern. Conversely, the aneurysm aortas exhibited disordered actin-positive cells, which represented fractured and disorganized VSMCs. The collagen of the aortic wall, which serves numerous essential functions for vessel homeostasis, revealed significant degeneration in the media of aneurysm aortas compared with controls (Figs. 1 and 2). 
A
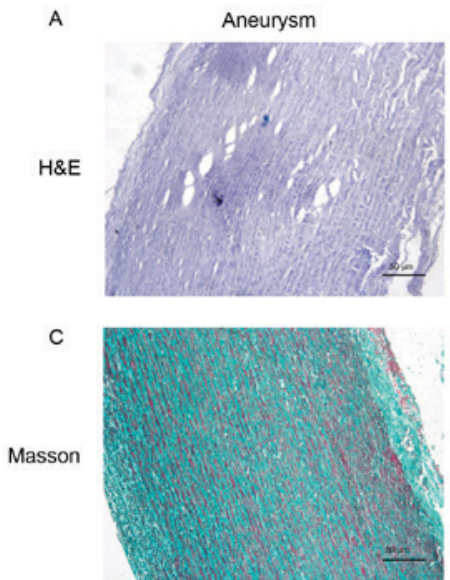
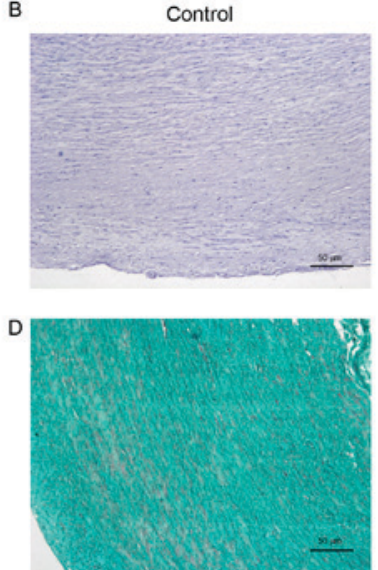

Figure 1. Morphological and structural alterations of VSCMs and collagen. Representative H\&E staining images of (A) aneurysm aorta with fractured and disordered VSMCs and (B) control sample, normal aorta. Representative Masson staining images of the collagen (blue-green or turquoise) in (C) aneurysm aorta and (D) control aorta. Magnification, x100. H\&E, Hematoxylin and Eosin; VSMC, vascular smooth muscle cells.
A

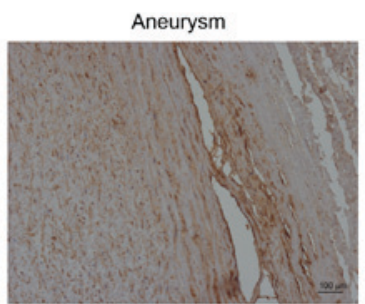

B

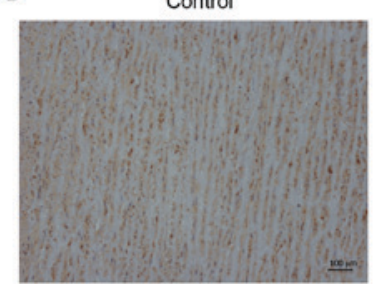

Figure 2. Immunostaining of SMA in the aneurysm samples and controls. (A) Fractured and disordered $\alpha$-SMA in the aneurysm samples. (B) Orderly pattern of immunoreactive $\alpha$-SMA in the controls. Magnification, $x 100$. $\alpha$-SMA, $\alpha$-smooth muscle actin.

Expression level of MMP-9 increases in AA. It has previously been demonstrated that increased MMP-9 results in VSMCs apoptosis and collagen degeneration in the most frequently occurring form of thoracic aortic aneurysm $(18,19)$. In the present study, the protein expression of MMP-9 was increased in the AA group compared with the control group, which was in accordance with results from the aforementioned previous studies. A total of 3 slices from each sample were randomly selected for evaluation. The final scores indicated increased expression of MMP-9 in the aneurysm aortas compared with the controls (Fig. 3).

Expression level of ADAMTS-7 increases in AA. ADAMTS-7 was primarily demonstrated to be located in the media and along the intima-medial border. Its expression was upregulated in the AA group compared with the control group. Analysis of the data collected from two independent observers suggested that the average score in the AA group was increased compared with controls (Fig. 4).

Expression level of COMP decreases in $A A$. COMP has previously been demonstrated to act as a normal component of the human artery wall, secreted by VSMCs $(10,11)$. Similar to ADAMTS-7, COMP was additionally primarily located in the media and along the intima-medial border.

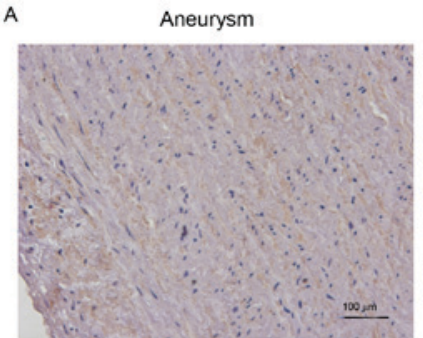

B Contro

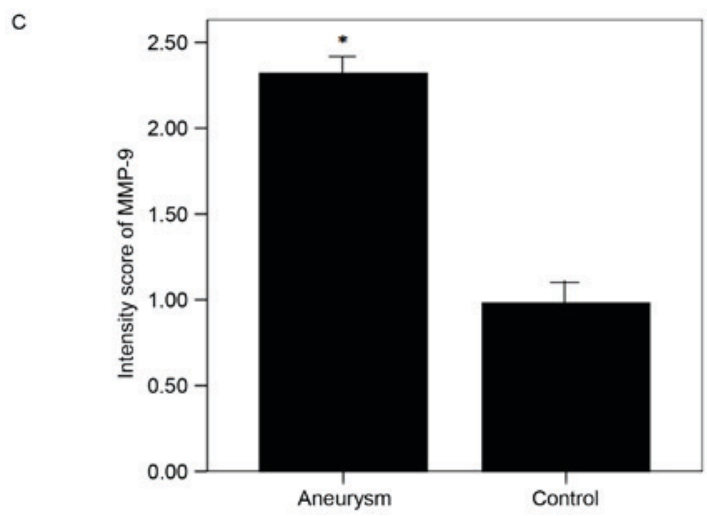

Figure 3. Protein expression levels of MMP-9 in the aneurysm aorta. (A) Aneurysm aorta and (B) control aorta representative images and (C) quantification of protein MMP-9 expression levels detected via immunohistochemistry. MMP-9 staining indicated by presence of brown chromogen. Semi-quantitative analysis demonstrated an increased expression of MMP-9 in aneurysm aortas $(n=4)$ compared with the control aortas $(n=6) .{ }^{*} P<0.05$ vs. control. Magnification, x400. MMP-9, matrix metalloproteinase-9.

Immunohistochemistry and western blotting analysis demonstrated that COMP expression was markedly decreased in the AA group compared with controls (Figs. 5 and 6).

\section{Discussion}

Research and exploration regarding the pathogenesis of aneurysm disease has evolved considerably over the past 30 years. It is widely believed that genetic influences, inflammation, calcification and atherosclerosis are the primary pathological inducers of AA. However, the exact underlying mechanism of its pathogenesis remains to be fully elucidated. An effective preventive therapeutic strategy against AA is still lacking. The present study analyzed ADAMTS-7 and COMP expression in human specimens, and suggests that the intervention of the interaction between these two factors may act as a novel therapeutic strategy in the future, for treatment of AA.

The ADAMTS proteinase family consists of 19 secreted, multidomain proteolytic enzymes and is important in various pathophysiological processes including ECM assembly and degradation, hemostasis, organogenesis, angiogenesis and development of genetic diseases, cancer and arthritis (20). ADAMTS-7 is a member of the ADAMTS family, which is identified as a novel locus associated with human and animal vascular atherosclerosis $(8,21-23)$. Bauer et al (8) reported that genetic deletion of ADAMTS-7 reduces neointima thickening following wire injury to the femoral and carotid arteries. Furthermore, Wang et al (10) suggested that ADAMTS-7 increases neointima formation in balloon-injured rat arteries by stimulating VSMC migration through the degradation of 
A

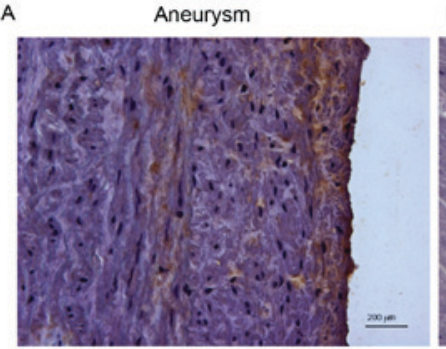

B

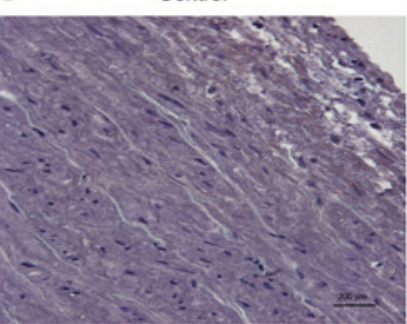

C

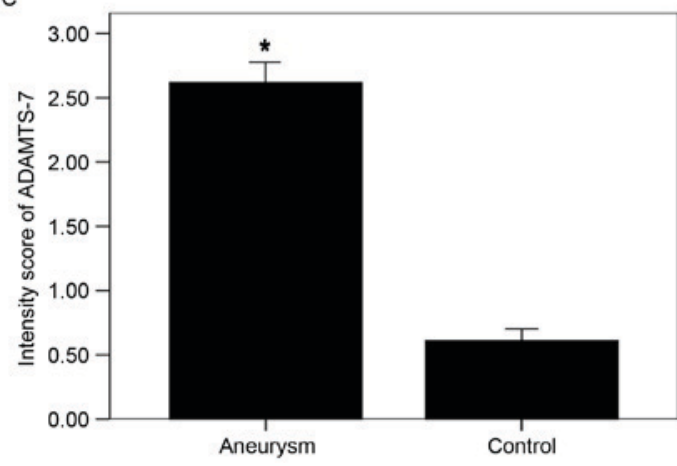

Figure 4. Protein expression levels of ADAMTS-7 in the aneurysm aorta. (A) Aneurysm aorta and (B) control aorta representative images and (C) quantification of protein ADAMTS-7 expression levels detected via immunohistochemistry. ADAMTS-7 was stained as brown chromogen which was primarily located in the media and along the intima-medial border. Semi-quantitative analysis demonstrated increased expression of ADAMTS-7 in aneurysm aortas $(n=4)$ compared with the control aortas $(n=6) .{ }^{*} \mathrm{P}<0.05$ vs. control. Magnification, $\mathrm{x} 400$. ADAMTS-7, a disintegrin and metalloproteinase with thrombospondin motifs 7 .
A

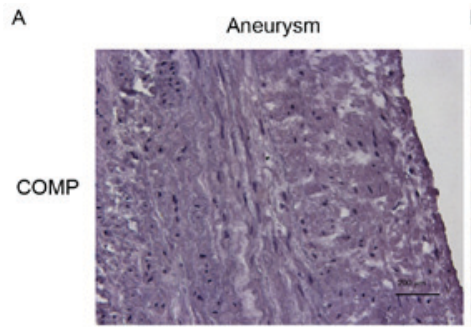

C

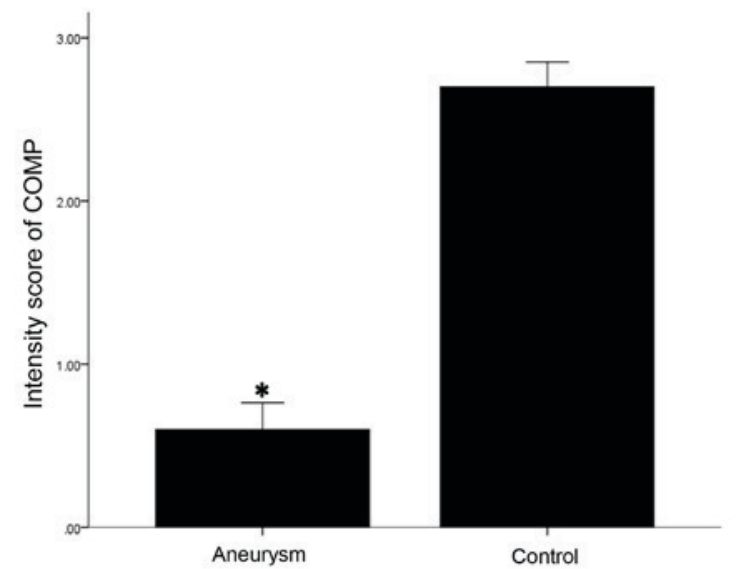

Figure 5. Protein expression of COMP in the aneurysm aorta. Representative images of COMP protein expression in (A) aneurysm and (B) control samples (magnification, x400; Scale bars, $200 \mu \mathrm{m}$ ). (C) Quantification of protein COMP expression levels detected via immunohistochemistry. COMP was stained as brown chromogen which was primarily located in the extracellular matrix and along the intima-medial border. Semi-quantitative analysis demonstrated that there was decreased expression of COMP in aneurysm aortas $(n=4)$ when compared with the control aortas $(n=6) .{ }^{*} \mathrm{P}<0.05$ vs. control. COMP, cartilage oligomeric matrix protein.

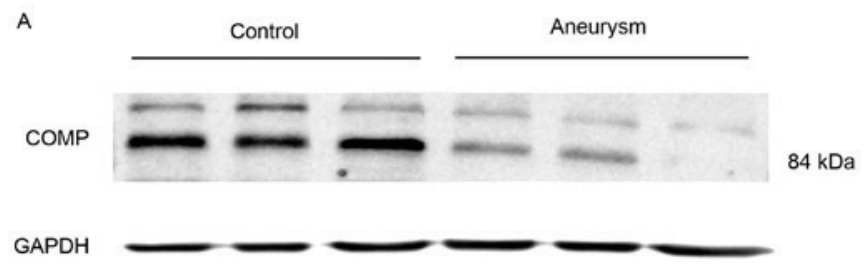

B

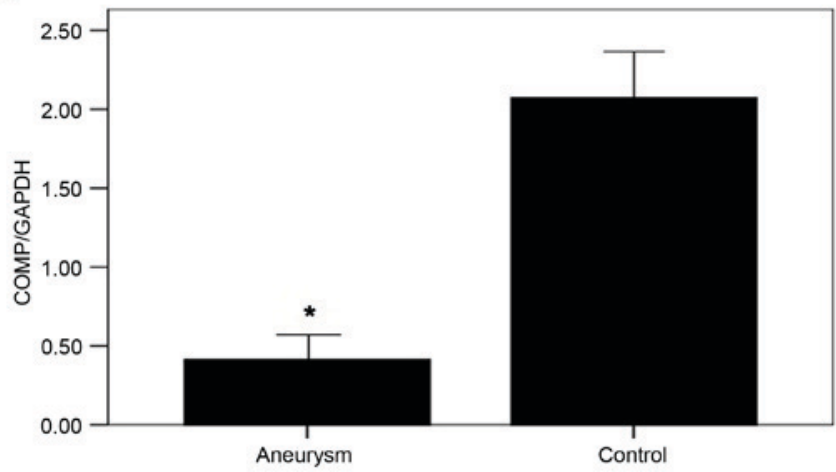

Figure 6. Representative image of (A) western blotting and (B) the semi-quantification of the COMP protein expression detected. Semi-quantitative analysis demonstrated that COMP expression was significantly decreased in the aneurysm aortas $(n=4)$ when compared with controls $(n=6)$, which is consistent with the results of immunohistochemistry. The results were normalized to those of GAPDH. ${ }^{*} \mathrm{P}<0.05$ vs. control. COMP, cartilage oligomeric matrix protein.

COMP. Du et al $(15,24)$ reported that silencing of ADAMTS-7 markedly reduces COMP degradation in calcifying rat VSMCs in vitro. Therefore, these findings suggest that ADAMTS-7 exhibits a critical role in intimal hyperplasia and atherosclerosis by stimulating VSMC migration through the degradation of COMP. However, to the best of the author's knowledge, there is no study currently available regarding the expression of ADAMTS-7 in human AA. The findings of the present study demonstrated increased expression of ADAMTS-7 in human AA.

In order to assess whether COMP was associated with the elevated ADAMTS-7 in human AA as described by previous studies in vascular atherosclerosis, the expression of COMP was evaluated by immunohistochemistry and western blotting analysis. The results revealed a reduced expression of COMP in human AA in the present study. Wang et al (10) reported that ADAMTS-7 facilitated vascular intimal hyperplasia via the degradation of COMP. In addition, Du et al (24) reported that knockdown of ADAMTS-7 attenuated COMP degradation. Therefore, ADAMTS-7 may be involved in the pathogenesis of vascular disorders via the degradation of COMP. In the present study, it was hypothesized that the downregulation of COMP was associated with the upregulation of ADAMTS-7 in human AA, while increased ADAMTS-7 may partially contribute to the pathogenesis of aortic aneurysm by decreasing COMP expression. COMP is a 524-kDa pentameric non-collagenous glycoprotein expressed in all types of cartilage, tendons and VSMCs (11). Previous studies identified COMP as a normal component of the artery wall, secreted by VSMCs $(11,14,25)$. The presence of COMP mRNA in different tissues was analyzed and it was revealed that the aorta is the only tissue outside the skeletal system in which COMP expression may 
be detected. COMP interacts with collagen type I, II and IX, proteoglycans, non-collagenous matrix proteins and is important in the stability of the collagen network (26-28). Furthermore, increased expression of COMP significantly suppresses VSMC migration (10). COMP is considered an inhibitor of ECM degradation and VSMC migration, which are characteristic symptoms of AA. The results of the present study indicated that upregulation of ADAMTS-7 may contribute to human AA by degradation of COMP.

ADAMTS-7 possesses mucin-proteoglycan domains and interacts with COMP via 4 C-terminal thrombospondin repeats (17). ADAMTS-7 may additionally bind directly to thrombospondin-1 (TSP-1) and is involved in endothelium repair (6). Therefore, it is important to identify the ADAMTS-7 cleavage sites in COMP and TSP-1 to define whether they are unique or shared with other metalloproteinase, and to identify if cleavage generates bioactive polypeptide fragments which bind cell receptors that trigger ECM and VSMC responses. ADAMTS-7 has previously been demonstrated to be a bioactive member of the ADAMTS family, therefore the search for other unique ADAMTS-7 substrates and interacting proteins in the artery wall may provide novel strategies for therapeutic intervention.

In conclusion, the present study observed the expression levels of ADAMTS-7 and COMP for the first time in human AA. The findings suggest that upregulation of ADAMTS-7 and down regulation of COMP are associated with human AA. Further studies are necessary to elucidate the potential mechanisms underlying the role of ADAMTS-7 and COMP in AA.

\section{References}

1. He R, Guo DC, Estrera AL, Safi HJ, Huynh TT, Yin Z, Cao SN, Lin J, Kurian T, Buja LM, et al: Characterization of the inflammatory and apoptotic cells in the aortas of patients with ascending thoracic aortic aneurysms and dissections. J Thorac Cardiovasc Surg 131: 671-678, 2006.

2. Ossovskaya VS and Bunnett NW: Protease-activated receptors: Contribution to physiology and disease. Physiol Rev 84: 579-621, 2004.

3. Spinale FG: Myocardial matrix remodeling and the matrix metalloproteinases: Influence on cardiac form and function. Physiol Rev 87: 1285-1342, 2007.

4. Aiken A and Khokha R: Unraveling metalloproteinase function in skeletal biology and disease using genetically altered mice. Biochim Biophys Acta 1803: 121-132, 2010.

5. Ashlin TG, Kwan AP and Ramji DP: Regulation of ADAMTS-1, -4 and -5 expression in human macrophages: Differential regulation by key cytokines implicated in atherosclerosis and novel synergism between TL1A and IL-17. Cytokine 64: 234-242, 2013.

6. Kessler T, Zhang L, Liu Z, Yin X, Huang Y, Wang Y,Fu Y, Mayr M, $\mathrm{Ge} \mathrm{Q}, \mathrm{Xu} \mathrm{Q}$, et al: ADAMTS-7 inhibits re-endothelialization of injured arteries and promotes vascular remodeling through cleavage of thrombospondin-1. Circulation 131: 1191-1201, 2015

7. Salter RC, Ashlin TG, Kwan AP and Ramji DP: ADAMTS proteases: Key roles in atherosclerosis? J Mol Med (Berl) 88: 1203-1211, 2010.

8. Bauer RC, Tohyama J, Cui J, Cheng L, Yang J, Zhang X, Ou K, Paschos GK, Zheng XL, Parmacek MS, et al: Knockout of Adamts7, a novel coronary artery disease locus in humans, reduces atherosclerosis in mice. Circulation 131: 1202-1213, 2015.

9. Reilly MP, Li M, He J, Ferguson JF, Stylianou IM, Mehta NN, Burnett MS, Devaney JM, Knouff CW, Thompson JR, et al: Identification of ADAMTS7 as a novel locus for coronary atherosclerosis and association of ABO with myocardial infarction in the presence of coronary atherosclerosis: Two genome-wide association studies. Lancet 377: 383-392, 2011.
10. Wang L, Zheng J, Bai X, Liu B, Liu CJ, Xu Q, Zhu Y, Wang N, Kong W and Wang X: ADAMTS-7 mediates vascular smooth muscle cell migration and neointima formation in balloon-injured rat arteries. Circ Res 104: 688-698, 2009.

11. Riessen R, Fenchel M, Chen H, Axel DI, Karsch KR and Lawler J: Cartilage oligomeric matrix protein (thrombospondin-5) is expressed by human vascular smooth muscle cells. Arterioscler Thromb Vasc Biol 21: 47-54, 2001.

12. Hedbom E, Antonsson P, Hjerpe A, Aeschlimann D, Paulsson M, Rosa-Pimentel E, Sommarin Y, Wendel M, Oldberg A and Heinegård D: Cartilagematrix proteins. An acidic oligomeric protein (COMP) detected in cartilage. J Biol Chem 267: 6132-6136, 1992.

13. Briggs MD, Hoffman SM, King LM, Olsen AS, Mohrenweiser H, Leroy JG, Mortier GR, Rimoin DL, Lachman RS, Gaines ES, et al: Pseudoachondroplasia and multiple epiphyseal dysplasia due to mutations in the cartilage oligomeric matrix protein gene. Nat Genet 10: 330-336, 1995.

14. Oldberg A, Antonsson P, Lindblom K and Heinegård D: COMP (cartilage oligomeric matrix protein) is structurally related to the thrombospondins. J Biol Chem 267: 22346-22350, 1992.

15. Du Y, Wang Y, Wang L, Liu B, Tian Q, Liu CJ, Zhang T, Xu Q, Zhu Y, Ake O, et al: Cartilage oligomeric matrix protein inhibits vascular smooth muscle calcification by interacting with bone morphogenetic protein-2. CircRes 108: 917-928, 2011.

16. Bond AR, Hultgårdh-Nilsson A, Knutsson A, Jackson CL and Rauch U: Cartilage oligomeric matrix protein (COMP) in murine brachiocephalic and carotid atherosclerotic lesions. Atherosclerosis 236: 366-372, 2014.

17. Rajkumar T, Stamp GW, Pandha HS, Waxman J and Gullick WJ: Expression of the type 1 tyrosine kinase growth factor receptors EGF receptor, c-erbB2 and c-erbB3 in bladder cancer. J Pathol 179: 381-385, 1996.

18. Rabskin SW: Differential expression of MMP-2, MMP-9 and TIMP proteins in thoracic aortic aneurysm-comparison with and without bicuspid aortic valve: A meta-analysis. Vasa 43: 433-442, 2014.

19. Wilson WR, Anderton M, Schwalbe EC, Jones JL, Furness PN, Bell PR and Thompson MM: Matrix metalloproteinase- 8 and -9 are increased at the site of abdominal aortic aneurysm rupture. Circulation 113: 438-445, 2006.

20. Wagstaff L, Kelwick R, Decock J and Edwards DR: The roles of ADAMTS metalloproteinases in tumorigenesis and metastasis. Front Biosci (Landmark Ed) 16: 1861-1872, 2011.

21. Patel RS and Ye S: ADAMTS7: A promising new therapeutic target in coronary heart disease. Expert OpinTher Targets 17: 863-867, 2013.

22. Hanby HA and Zheng XL: Biochemistry and physiological functions of ADAMTS7 metalloprotease. Adv Biochem: 1,2013.

23. You L, Tan L, Liu L, Shen R, Chaugai S, Wang DW and Cui W: ADAMTS7 locus confers high cross-race risk for development of coronary atheromatous plaque. Mol Genet Genomics 291: 121-128, 2016.

24. Du Y, Gao C, Liu Z, Wang L, Liu B, He F, Zhang T, Wang Y, Wang X, Xu M, et al: Upregulation of a disintegrin and metalloproteinase with thrombospondin motifs- 7 by miR-29 repression mediates vascular smooth muscle calcification. Arterioscler Thromb Vasc Biol 32: 2580-2588, 2012.

25. Wang L, Zheng J, Du Y, Huang Y, Li J, Liu B, Liu CJ, Zhu Y, Gao Y, Xu Q, et al: Cartilage oligomeric matrix protein maintains the contractile phenotype of vascular smooth muscle cells by interacting with alpha(7)beta(1) integrin. Circ Res 106: 514-525, 2010.

26. Thur J, Rosenberg K, Nitsche DP, Pihlajamaa T, Ala-Kokko L, Heinegård D, Paulsson $\mathrm{M}$ and Maurer P: Mutations in cartilage oligomericmatrixprotein causing pseudoachondroplasia and multiple epiphyseal dysplasia affect binding of calcium and collagen I, II and IX. J Biol Chem 276: 6083-6092, 2001.

27. Mann HH, Ozbek S, Engel J, Paulsson M and Wagener R: Interactions between the cartilage oligomeric matrix protein and matrilins. Implications for matrix assembly and the pathogenesis of chondrodysplasias. J Biol Chem 279: 25294-25298, 2004.

28. Halász K, Kassner A, Mörgelin M and Heinegård D: COMP acts as a catalyst in collagen fibrillogenesis. J Biol Chem 282: 31166-31173, 2007. 